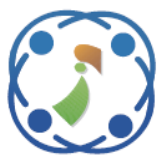

\title{
Feature Selection based on EEMD-LaS and Optimized BSO-LSSVM Classifier Model in Bearing Fault Diagnosis
}

\author{
VietHung Nguyen ${ }^{1 *}$ \\ DinhToan Nguyen ${ }^{1}$ \\ NhatTan Nguyen ${ }^{2}$ \\ ${ }^{1}$ Faculty of Mechanical Engineering, Hanoi University of Industry, Hanoi, Vietnam \\ ${ }^{2}$ VietNam-Japan Center of Mechanical Engineering, Hanoi University of Industry, Hanoi, Vietnam \\ *Corresponding author's Email: hung2009haui@gmail.com; nguyenviethung@ haui.edu.vn
}

\begin{abstract}
The trend of Industry 4.0 development in the maintenance field requires the big data analysis of system operation for predicting, or identifying any possible problem. This study proposed an automatic fault diagnosis method for rolling bearing. Ensemble empirical mode decomposition (EEMD) is used to preprocess the vibration signals of rolling bearing which solved the problem of mode mixing existing in empirical mode decomposition (EMD). The features are then extracted from the first IMFs in the time and frequency domain to form an initial fault feature of the complex vibration signal. The Laplacian score (LaS) method for selecting features is then utilized to construct a new sensitive feature according to their significances, named EEMD-LaS. An optimized pattern classification method is constructed to achieve an automatic diagnosis of bearing fault. This method uses a backtracking search optimal (BSO) algorithm to optimize the parameters of the least-squares support vector machine (LSSVM) classifier model, named BSO-LSSVM. Finally, the EEMD-LaS and BSO-LSSVM base proposed method for bearing fault diagnosis is applied to analyze the experimental data. The result indicates that the proposed EEMD-LaS - BSO-LSSVM method can effectively diagnose the fault categories with $100 \%$ accuracy of the outer-race fault and normal bearing condition; and 97\% minimal accuracy of the severe conditions of the inner-race fault and roller fault of rolling bearing.
\end{abstract}

Keywords: Bearing fault, Feature selection, Diagnosis, Laplacian score (LaS), Big data, Optimized classifier model.

\section{Nomenclature}

EEMD Ensemble empirical mode decomposition

IMF Intrinsic mode function

LaS Laplacian score

EEMD-LaS The extracted sensitive features

BSO Backtracking search optimal (BSO) algorithm

LSSVM Least-squares support vector machine

BSO-LSSVM Optimized classification model

$x(t) \quad$ Vibration signal in time-domain.

$y_{i}$ Objective class

$g$ Ameliorative evaluation function

$(\gamma, \sigma)$ The parameter pair of LSSVM classifier model.

RMS Root mean square

$S$ Skewness

$S D \quad$ Standard deviation

$K$ Kurtosis
CF Crest factor

(IF) Impulse factor

(SE) Shanon entropy

\section{Introduction}

The bearing is an important part of the rotating machinery. Any failure of a bearing may cause inefficient operation and unsafe conditions for the operator. Hence, the fault diagnosis method in the mechanical maintenance field is a focus of researchers. Normally, the diagnosis method can be summarized into several consecutive stages as (1) acquisition vibration data, (2) signal processing, (3) feature extraction and selection, and (4) pattern classification. A good method can diagnose exactly the fault status even the initial acquired data includes the complex chaos of noise.

The pattern feature normally plays an important role in the fault diagnosis. These features need to be 
extracted from the original vibration signal, which is often referred to as non-linear, non-station data. Recently, time-frequency domain-based analysis methods have been effectively applied to extract the important feature. Ensemble empirical mode decomposition (EEMD) is a powerful adaptive analysis method of non-linear and non-stationary data $[1,2]$. EEMD is proposed base on empirical mode decomposition (EMD) to take the advantage of the filter bank behavior of the EMD and to alleviate the mode mixing problem of the EMD method. EEMD decomposes the original signal into intrinsic mode functions (IMFs) over an ensemble of white noisy copies. EEMD has been successfully applied to different fields, such as fault diagnosis, earthquake, climate, etc [3-5]. In this study, the first IMFs of EEMD are used to extract the feature in time and frequency domains which are formed as the initial high-dimensional feature matrix. To obtain the bearing fault diagnosis task, this initial feature matrix is difficult to distinguish the most important features. In fact, not all values in which are related with the fault information. So, unrelated features will cause the training process time-consuming and even accurate decreasing for diagnosis phase [4]. Therefore, the feature selection is needed during the initial high-dimensional feature matrix. The modern method of feature selection mainly includes HilbertSchmidt Independence Criterion (HSIC) [6], Laplacian Score(LS) [7, 8], Principal Component Analysis (PCA) [9], and so on. The Laplacian Score is a classic filter unsupervised feature selection algorithm and it not only considers the importance of features but also fully considers the correlation between features. Compared with other methods, the LS is the most prominent method which is introduced by X. He et al. [7] and X. Y. Jing et al. [10]. The methodology of the method is to rank the elements in features from low scores to high ones according to their significances. In this bearing fault diagnosis task, LS is applied to the initial feature matrix of the EEMD to exploit the new sensitive feature matrix of bearing fault, named EEMD-LaS, which can reduce the training time and improve the efficiency of the fault diagnosis model.

The least-squares support vector machine (LSSVM) proposed by Suyken is based on statistical learning theory and structural risk minimization principle [11]. LSSVM is the improvement of standard support vector machine (SVM), which is a powerful machine learning tool for reducing the computational complexity that has been employed in applications such as pattern recognition, time-series forecasting, robotics, and diagnostics [12-16]. LSSVM takes the equality constraints to replace the inequality constraints, selects the least-square linear system as the loss function, and transforms the solution of quadratic programming into solving linear equations. The performance of LS-SVM heavily depends upon the selection of its two parameters, the penalty factor and kernel parameter. These parameters affects the classification accuracy of the model, whereas the theoretical basis of selecting appropriate parameters for LS-SVM is still lacking $[17,18]$. Therefore, the parameter pair is required the optimal values in the architectural design. To lift the restriction of the parameter selection, the backtracking search optimal (BSO) algorithm is applied for selecting the optimal parameters of the LSSVM classification model, named BSO-LSSVM. BSO algorithm is an evolutionary algorithm (EA) that has been developed by Civicioglu [19] which uses three operators of selection, mutation, and crossover to solve problems of real-valued numerical optimization.In this study, an optimized BSOLSSVM classifier model is constructed for the intelligent fault diagnosis of rolling bearing. Based on the EEMD-LaS and BSO-LSSVM, an intelligent fault diagnosis method for the rolling bearing is proposed. Firstly, the EEMD method is used to decompose the bearing vibration signals into IMFs and a residue. Secondly, the features are extracted in time and frequency domains from the first several IMFs. Thirdly, the LaS for feature selection is used to rank the extracted features according to their significances. The new sensitive EEMD-LaS fault features are then constructed according to the ranked results that serve the classifier model with input. Finally, the optimized BSO-LSSVM based classifier model is constructed to accomplish the diagnosis object of bearing fault.

The organization of the rest of the paper is presented as follows: Section 2 presents the proposed method based on EEMD-Las for extracting feature and select. Section 3 presents construction of the optimized classifier model based on BSO algorithm and the basic LSSVM classifier. Section 4 presents the experimental result for bearing fault diagnosis where the results are compared and analyzed with the other methods. Section 5 is the conclusions. Acknowledgments and a list of references then follow.

\section{Proposed EEMD-LaS method for extracting and selecting feature}

\subsection{Empirical mode decomposition (EMD) and ensemble EMD (EEMD)}


EMD is an interesting method that has been applied effectively for analyzing the complex data of non-stationary, non-linear [20]. The EMD method is used to decompose a signal automatically into a set of band-limited functions, intrinsic mode functions (IMF). These functions are estimated by an iterative procedure called sifting. Each IMF should satisfy two basic conditions: (1) the number of extreme points and the number of zero crossings must either be equal or differ by one at most in the whole data; and (2) at every point, the mean value of the envelopes defined by local maxima and local minima is zero. The EMD can separate a segment of a vibration signal $x(t)$ into $n$ IMFs: $\left\{I M F_{1}, I M F_{2}, \ldots, I M F_{n}\right\}$, and a residue signal $r$. Hence, $x(t)$ can be reconstructed as a linear combination:

$$
x(t)=\sum_{n=1}^{N} I M F_{n}+r
$$

EMD is a sifting process and is a systematic way to extract IMFs. The procedure of EMD algorithm can be described as follows:

Given an input signal $x(t), r(t)=x(t), n=0$.

Step 1: Get the local maximum and local minimum of $x(t)$
Step 2: Get the upper envelope $e_{\max }(t)$ by connecting all local maxima through cubic spline functions. Repeat the procedure for the local minima to produce the lower envelope $e_{\min }(t)$.

Step 3: Calculate the mean value at every point of the envelopes:

$$
m(t)=\frac{1}{2}\left(e_{\max }(t)+e_{\min }(t)\right)
$$

Step 4: Calculate $h(t)=x(t)-m(t)$. If $h(t)$ satisfies the IMF condition, then $n=n+1, I M F_{n}=$ $h(t)$ and go to Step 5; else $x(t)=h(t)$, go to Step 1 .

Step 5: Let $r(t)=r(t)-I M F_{n}$. If $r(t)$ is a monotonic function, end the sifting process, else $x(t)=r(t)$ and go back to Step 1 .

Ensemble EMD (EEMD) method was developed by $\mathrm{Wu}$ and Huang $[1,2]$ to eliminate the mode mixing problem in the decomposing complex signal. EEMD is based on the dyadic property of the EMD method and the addition of the white noise in the data analysis. EEMD can clearly separate the natural scale of signals and produce IMFs with full physical meaning. The provision of more credible IMFs than the

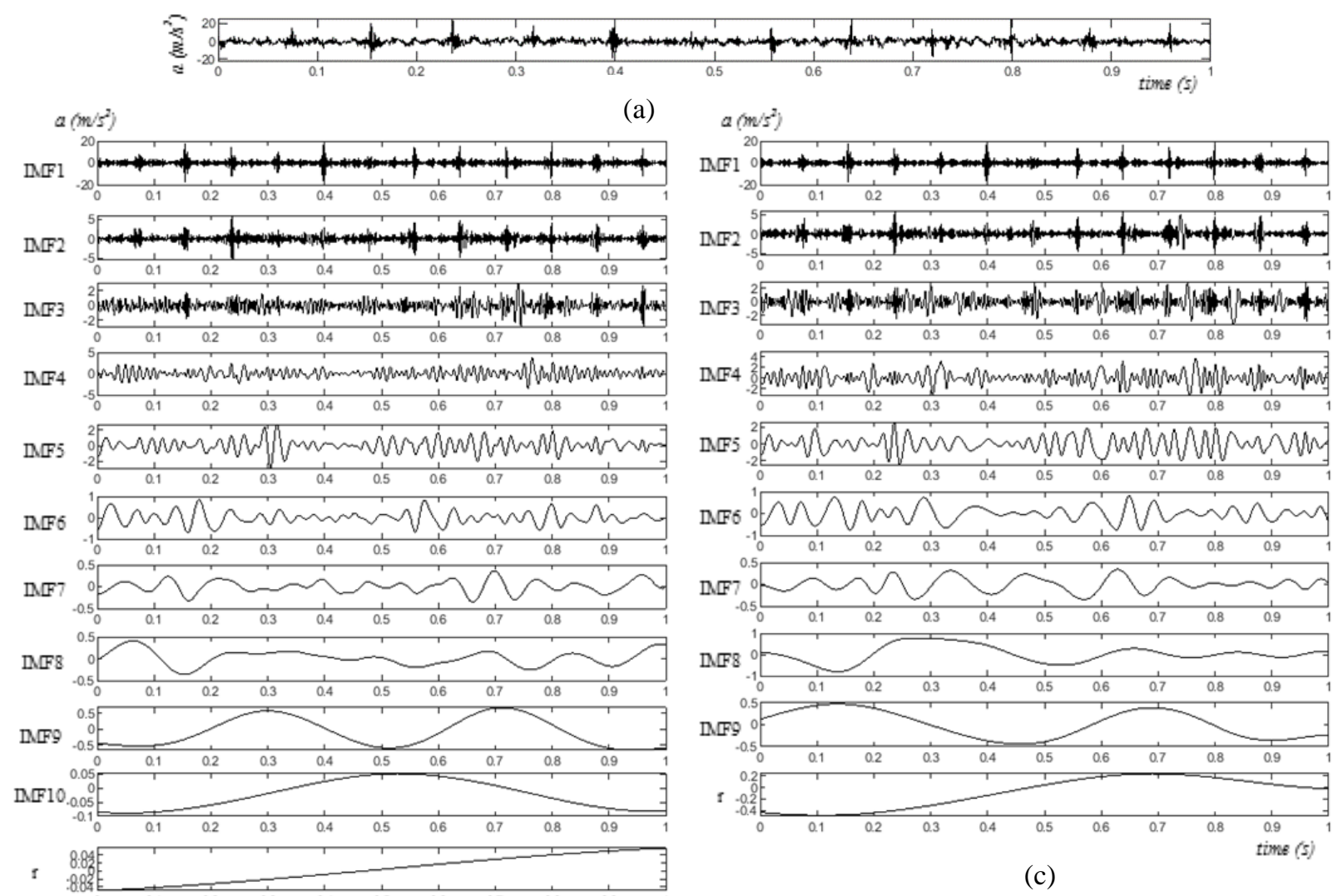

(b)

Figure. 1 A sample of vibration signal is decomposed by EEMD and EMD: (a) Original vibration signal, (b) Using EEMD and (c) Using EMD 
previous EMD method is an advantage for analyzing the complex data in its superiority and application [20, 21]. The principle advanced in EEMD can be crucially described as follows:

Step 1: Add the white noise $w_{i}(t), i=1,2, \ldots, N$ to the given signal, $n_{i}(t)=x(t)+w_{i}(t)$.

Step 2: Decompose the noisy signal $n_{i}(t)$ to get IMFs, $I M F_{i, j}(t), j=1,2, \ldots, K$ by using EMD algorithm.

Step 3: Increase i value and repeat the step 1 and 2 with the number of trials $\mathrm{N}$.

Step 4: Calculate the final IMFs after the number of trials, $\mathrm{N}$, as $I M F_{i, j}=\frac{1}{N} \sum_{i=1}^{N} I M F_{i, j}(t)$.

Fig. 1 shows the effective of the both EMD and EEMD methods. Both EMD and EEMD method can decompose a complex vibration signal, bearing outerrace fault, into a series of intrinsic mode functions (IMFs). Each of the IMFs includes different frequency bands ranging from high-to-low stationary and implies a distinct time characteristic scale. Observed that EEMD method is better than the EMD method, EEMD can still decompose the data effectively and accurately when the EMD is invalid. The noise-assisted analysis EEMD method can both suppress the mode mixing effectively and improve the accuracy of decomposition. In this study, authors used EEMD to extract significant features in complex vibration signals of bearing, which were based on the first several IMFs [3].

Table 1. The features of vibration signal

\section{Features} Mathematical Equation

Root mean square $(R M S) \quad R M S=\sqrt{\frac{1}{N} \sum_{i=1}^{N} x_{i}^{2}}$

where $x$ is vibration data, $x=\left\{x_{1}, x_{2}, \ldots, x_{n}\right\}$

Skewness $(S)$

$$
S=\frac{1}{(N-1) S D^{3}} \sum_{i=1}^{N}\left(x_{i}-\bar{x}\right)^{3}
$$

where:

$S D$ is standard deviation of vibration signal $S D=\sqrt{\frac{1}{N-1} \sum_{i=1}^{N}\left(x_{i}-\bar{x}\right)^{2}}$ and $\bar{x}=\frac{1}{N} \sum_{i=1}^{N} x_{i}$

Kurtosis $(K)$

$$
K=\frac{1}{(N-1) S D^{4}} \sum_{i=1}^{N}\left(x_{i}-\bar{x}\right)^{4}
$$

Crest factor $(C F)$

$$
C F=\frac{\max (x)}{\sqrt{\frac{1}{N} \sum_{i=1}^{N} x_{i}^{2}}}
$$

$$
\text { Impulse factor }(I F) \quad I F=\frac{\max (x)}{\bar{x}}
$$

Shanon entropy $(S E)$

where:

$$
S E=-\sum_{(k)} P_{k} \log P_{k}
$$

$P_{k}$ is the energy probability distribution defined as $P_{k}=\left|t_{i}\right|^{2} / \sum_{i=1}^{n}\left|t_{i}\right|^{2}$;

$t_{i}$ is the spectrum of $x(i)$ vibration signal, $i=1,2, \ldots, n, n$ is the number of spectrum lines. 
Table 2. Construction of the initial features of vibration signal

\begin{tabular}{llllllllll}
\hline 1 & $R M S_{1}$ & 7 & $R M S_{2}$ & 13 & $R M S_{3}$ & 19 & $R M S_{4}$ & 25 & $R M S_{5}$ \\
2 & $S_{1}$ & 8 & $S_{2}$ & 14 & $S_{3}$ & 20 & $S_{4}$ & 26 & $S_{5}$ \\
3 & $K_{1}$ & 9 & $K_{2}$ & 15 & $K_{3}$ & 21 & $K_{4}$ & 27 & $K_{5}$ \\
4 & $I F_{1}$ & 10 & $I F_{2}$ & 16 & $I F_{3}$ & 22 & $I F_{4}$ & 28 & $I F_{5}$ \\
5 & $C F_{1}$ & 11 & $C F_{2}$ & 17 & $C F_{3}$ & 23 & $C F_{4}$ & 29 & $C F_{5}$ \\
6 & $S E_{1}$ & 12 & $S E_{2}$ & 18 & $S E_{3}$ & 24 & $S E_{4}$ & 30 & $S E_{5}$ \\
\hline
\end{tabular}

\subsection{Feature extraction and selection based on EEMD-LaS}

A pattern feature set is needing for the performing fault diagnosis effectively. To fully represents the fault information of original signal, first five IMFs are used. We extract features in time-domain and in frequency-domain of each IMF, which includes root mean square $(R M S)$, skewness $(S)$, Kurtosis $(K)$, impulse factor $(I F)$, crest factor $(C F)$ and shannon entropy (SE), respectively. The features are defined in Table 1. These extracted features form the highdimensionality vector, named $F$ vector. The obtained features, $E=\left\{E_{1}, E_{2}, \ldots, E_{6}\right\} \quad$, where $E_{i}=$ $[R M S, S, K, I F, C F, S E]$ includes owns 30 values of each vibration signal as show in Table 2 . This feature $F$ vector is ideally able to represent the fault information in different views. However, not all values in which are related with the fault information. So, unrelated features will cause the training process time-consuming and even accurate decreasing for fault diagnosis. It is necessary to select the most interrelated feature with fault information from the 30 features. In this study, $\mathrm{LaS}$ is employed to select the most important features from the initial fault features, named EEMD-LaS. LaS is fundamentally proposed based on the Laplacian eigenmaps and locality preserving projection [7]. The selected EEMD-LaS features are the most important features from the initial $E$ feature vector for reflecting the fault information. These features are resorted according to their locality preserving power from low to high. After that the obtainer EEMD-LaS features of lowerdimensions vector serves the claasifier model with input. A classifier model base on LSSVM suitable for performing the classification problems of fault mode. This model can be select the optimal parameters by the evolutionary algorithm, BSO algorithm. the obtained classifier model is then used to automatically identify fault category and severity.

\section{Optimal machine learning model based on LSSVM}

The machining learning model is the important stage of diagnosis process. The LSSVM classifier model is used as basic classifier with the parameters is optimized by the BSO algorithm, BSO-LSSVM. The optimed BSO-LSSVM classifier model, used the EEMD-LaS feature data to train and evaluate.

\subsection{LSSVM classification model}

LSSVM was proposed by Suykens in 1999 [11] to diminish the computational burden of SVM, which applies with equality instead of inequality constraints. LSSVM has solved the following convex optimization problems with good generalization ability which try to find the optimal separating hyperplane. Suppose $\left\{\left(x_{i}, y_{i}\right) \mid i=1,2, \ldots, l\right\}$ as training set of sample number is $l$. The sample of $x_{i}, i=1,2, \ldots, l$ corresponds to the category of $y_{i} \in$ $(-1,1)$, the objective function and constraint condition are shown as follows:

$$
\left\{\begin{array}{c}
\min \left(J_{L S}\right) ; J_{L S}(w, e)=\frac{1}{2} w^{T} w+\frac{1}{2} \gamma \sum_{i=1}^{l} e_{i}^{2} \\
\text { s.t. } y_{i}\left(w^{T} \varphi\left(x_{i}\right)+b\right)=1-e_{i}, i=1,2, \ldots, l
\end{array}\right.
$$

where $e_{i}$ are slack variables and $\gamma \geq 0$ is a penalty factor/ regularization parameter.

The values of $\gamma$ will influence training result of the LSSVM model. The low value of $\gamma$ means a model with high training errors and oppositely the high $\gamma$ value does not permit any slack variables and consequently increases model complicity. Therefore, it is critical to find proper value for $\gamma$ and it is one of LSSVM tuning parameter that should be adjusted conscientiously.

Define Lagrange function:

$$
\begin{aligned}
& L(w, b, e, \alpha)=\frac{1}{2} w^{T} w+\frac{1}{2} \gamma \sum_{i=1}^{l} e_{i}^{2}- \\
& -\sum_{i=1}^{l} \alpha_{i}\left\{y_{i}\left(w^{T} \varphi\left(x_{i}\right)+b\right)+e_{i}-1\right\}
\end{aligned}
$$

where $\alpha_{i}$ is Lagrange multiplier, which can be positive or negative. The optimum condition is as follows:

$$
\frac{\partial L}{\partial w}=0, \frac{\partial L}{\partial b}=0, \frac{\partial L}{\partial e_{i}}=0, \frac{\partial L}{\partial \alpha_{i}}=0
$$

The matrix equation can be obtained easily as follows: 


$$
\left[\begin{array}{cc}
0 & Y^{T} \\
Y & \Omega+l / \gamma
\end{array}\right]\left[\begin{array}{l}
b \\
\alpha
\end{array}\right]=\left[\begin{array}{l}
0 \\
\bar{I}
\end{array}\right]
$$

where $Y^{T}=\left[y_{1}, y_{2}, \ldots, y_{l}\right] ; I$ is unit matrix $\bar{I}=$ $[1,2, \ldots, l]^{T} \quad ; \quad \alpha=\left[\alpha_{1}, \alpha_{2}, \ldots, \alpha_{l}\right]^{T} ; \quad \Omega=$ $y_{i} y_{j} \varphi^{T}\left(x_{i}\right) \varphi\left(x_{j}\right)=y_{i} y_{j} K\left(x_{i}, x_{j}\right) ; K\left(x_{i}, x_{j}\right)$ is Kernel function of SVM.

The classifying function of LSSVM classifier finally can be obtained as follows:

$$
f(x)=\operatorname{sgn}\left(\sum_{i=1}^{l} \alpha_{i} y_{i} K\left(x, x_{i}\right)+b\right)
$$

In this research, Gauss radial basic function (RBF) is utilized to map the samples into a higher dimension feature space which can make LSSVM get better performance and generalization, as shown in Eq. (14).

$$
K(x, y)=\exp \left(-\frac{\|x-y\|^{2}}{2 \sigma^{2}}\right)
$$

In summary, the classification effect of LSSVM is controlled by the parameter pair $(\gamma, \sigma)$, where the penalty factor parameter $\gamma$ is mentioned in Eq. (10) and $\sigma$ is a Kernel parameter mentioned in Eq. (14). The appropriate selection of two parameters will affect the learning performance of LSSVM. Therefore, this parameter pair values must be optimized to obtain an effective classification accuracy, which can be done by the BSO algorithm.

\subsection{Backtracking Search Optimization (BSO) algorithm}

BSO algorithm is a new meta-heuristic algorithm in the evolutionary series [19]. BSO uses information obtained from past generations to search for better fitness solutions. The general structure of BSO is presented as below. The bio-inspired philosophy of BSO is correspondent with a social group of living creatures, which relate to hunting areas for finding food at random movements.

BSO start
Initialization
$\quad$ Repeat
Selection I
Generate new population:
Mutation
Crossover
$\quad$ End

\section{Selection II}

Until Stopping conditions are met

Initialization phase: the algorithm generates and evaluates the initial population $P$ and starts a historical population $P_{o}$. The historical population constitutes the memory of BSOA.

Selection I: the algorithm randomly determines whether the current population $P$ is recorded as the historical population $P_{o}$. The individuals of $P_{o}$ are then shuffled.

Mutation: the mutation $P_{m}$ operator is created by an initial version of the new population $P_{n}$, according to Eq. (15). $P_{m}$ is the result of the individuals movement $\left(P_{o}-P\right)$ and the motion amplitude $F=$ $k \cdot r_{\text {rand }}$. In which the directions set by. And, defines, and is given by Eq. (15).

$$
P_{m}=P_{n}+F\left(P_{o}-P\right)
$$

where $k$ value is adjusted empirically during prior simulations; $r_{\text {rand }} \sim N(0,1), N$ is the standard normal distribution.

Crossover: The operator randomly crosses over elements from $P_{m}$ with elements of $P$, which generates the final version of $P_{n}$.

Selection II: the algorithm selects elements from $P_{n}$ to have better fitness than elements of $P$. And then $P_{n}$ replaces them in $P$. Thus, $P$ only receives new evolved individuals. After meeting the stopping conditions, the algorithm returns the best solution found.

\subsection{Optimized BSO-LSSVM model}

Two parameters $(\gamma, \sigma)$, parameter pair of LSSVM are important in constructing the classifier model which may be obtained by using optimization algorithm. BSOA algorithm is used to explore the search space of the given LSSVM classification problem to seek out the parameters $(\gamma, \sigma)$ required to maximise the actual objective of accuracy [22]. The principled training phase of the optimal BSOLSSVM classifier model includes several main steps, which is implemented as follows:

Step 1: Prepare the input data.

Step 2: Initiate the LSSVM with the random parameters $\gamma$ and $\sigma$. Set iterative variable $t=0$ and perform the training process for next steps. $t+1$

Step 3: Increase the iteration variable by set $t=$

Step 4: Evaluate the amelioration by the $g=$ $\sqrt{\frac{1}{N} \sum_{(i)}\left(y_{i}-y_{T i}\right)^{2}}$. This $g$ function is used to evaluate the quality of every element. Where $y_{T}$ is the right classified samples, and $N$ is summed the samples in the testing data. The desirable value is one to best classification accuracy.

Step 5: Stop criteria checking. The deterioration 


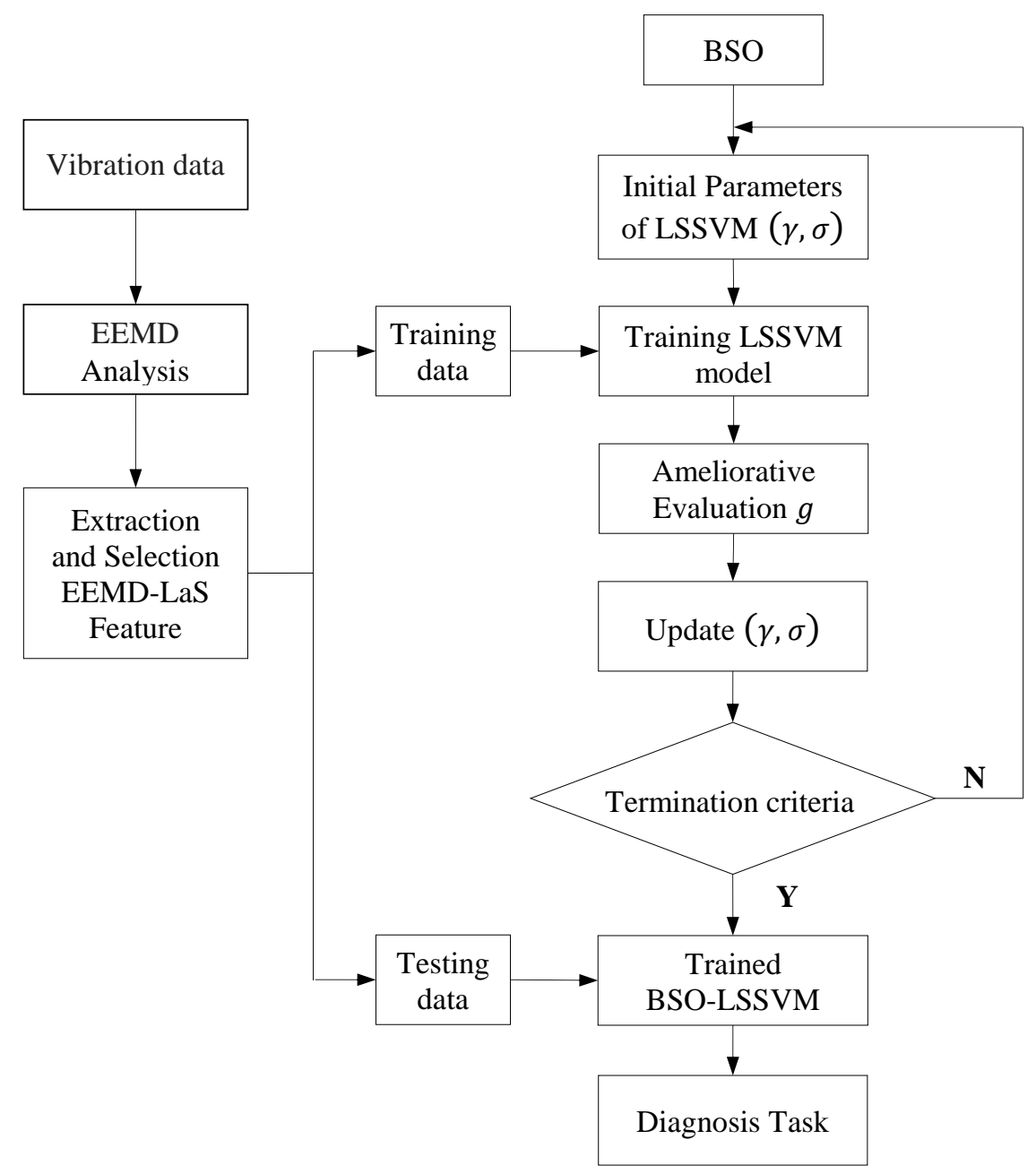

Figure. 2 Flowchart of the proposed EEMD-LaS - BSO-LSSVM method

function is satisfied by $\mathrm{g}$ function or iteration is maximal go to Step 7. If not, go to next step.

Step 6: Update the new $\gamma, \sigma$ parameters, going to Step 3.

Step 7: End of training procedure, the trained BSO-LSSVM classifier model is optimized.

The efficient search capability of BSO algorithm generated the optimal $(\gamma, \sigma)$ parameter pair to LSSVM classifier. The optimized BSO-LSSVM can bring out the high classification accuracy.

\section{Proposed diagnosis method of bearing fault}

\subsection{EEMD-LaS-BSO-LSSVM proposed method}

based

The EEMD-LaS-BSO-LSSVM proposed method is formed including two main stages: (1) EEMD-LaS selection method is to extract the feature of gear fault vibration data and (2) BSO-LSSVM is the classification model. EEMD-LS method is used to exploited the features of original vibration signal that mean for increasing the overall reliability and identification accuracy of the classification procedure. The optimized BSO-LSSVM classifier models is constructed to accurately identify the fault statuse of normal bearing (NB), inner-race fault (IF), outer-race fault (OF) and roller fault (RF), respectively. This technique can be visualized by the flowchart in Fig. 2, which can be described as follows: As

Step 1: Collect the vibration data of the testing bearing conditions.

Step 2: Use the EEMD to decompose data into the IMFs.

Step 3: Extract the feature from the first IMFs to construct the initial $\mathrm{F}$ feature matrix

Step 4: Select the feature using LaS to generate the sensitive EEMD-LaS feature. And, divide the feature data in to a training-testing partition.

Step 5: Train and test the BSO-LSSVM classification model to identify the actual bearing fault. Bearing fault EEMD-LaS-BSO-LSSVM diagnostic technique demonstrated diagnostic accuracy and reliability in experiments. 

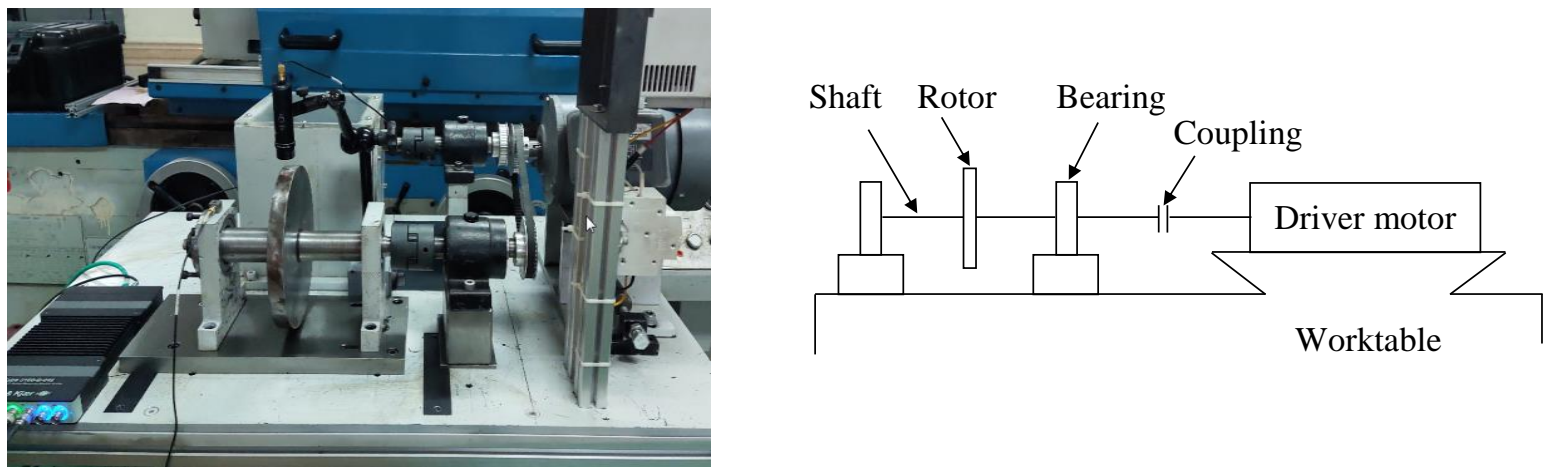

Figure. 3 Test rig of the experimental setup

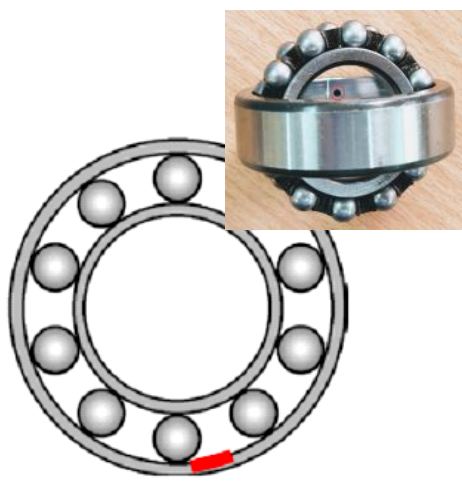

(a)

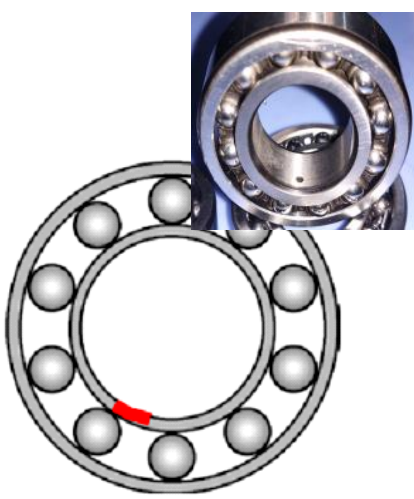

(b)

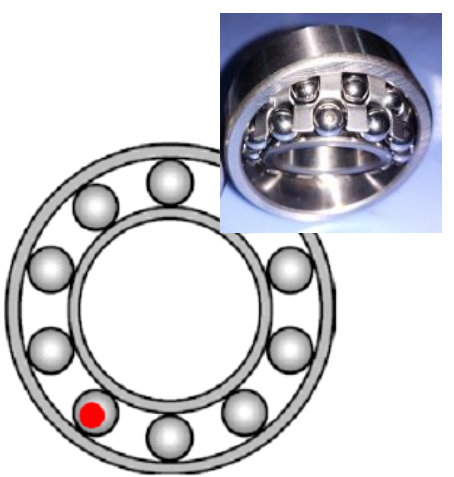

(c)

Figure. 4 Schematic of bearing fault conditions: (a) OF status, (b) IF status and (c) RF status

\subsection{Experimental analysis}

To evaluate the proposed diagnosis technique, we use a test rig shown in Fig. 3 to generate the experimental vibration data. A rotor and two test roller bearings of 1205-type are installed on the shaft. Its grouping in four bearing conditions are tested for NB, IF, OF and RF separately. Fig. 4 show the test conditions of roller bearing. The test bearings are installed on motor shaft where is loaded by the dynamometer. An acceleration sensor of Triaxial Delta Tron 4525B-001 type (Bruel\&Kjær) has been fixed on the bearing house. The test bearing is placed DE with the sized defects in $2.5 \mathrm{~mm}$ fault diameter and $1.5 \mathrm{~mm}$ fault depth are generated by the machining of electro-discharge approach, and shaft speed is 1512 RPM. The acquired vibration signals at $10 \mathrm{kHz}$ of sampling frequency are grouped in eighty signals that are then divided into training and testing data as show in Table 3. Fig. 5 shows a vibration signal sample within the time domain with three tested gear faults, which can imply that the vibration intensity is unclear can't be determined albeit the loading parameter is unchanged.

\subsection{Results and discussion}

As described in Table 3 there are four classes of samples under our consideration. In each class 15 samples are selected for training the classifier so that totally 60 samples. Firstly, the EEMD method is used to decompose the vibration signal of each class with several IMFs obtained. Fault information locates generally in the high frequency components, the features of the first five IMFs in time and frequency domains are extracted (shown in Table 1). Therefore, each training sample can be represented by a vector with 30 values called initial fault feature. The 60 initial fault features are used to construct the initial fault feature matrix $F(60 \times 30)$. Secondly, the LaS of the 30 features in $F$ are computed and sorted from low score to high ones. Then the first five features

Table 3. Collection of vibration signal samples

\begin{tabular}{lccc}
\hline Testing status & Class & $\begin{array}{c}\text { Collecting } \\
\text { sample }\end{array}$ & $\begin{array}{c}\text { Training } \\
\text { samples }\end{array}$ \\
\hline Normal bearing (NB) & 1 & 20 & 15 \\
Inner-race fault (IF) & 2 & 20 & 15 \\
Outer-race fault (OF) & 3 & 20 & 15 \\
Roller fault (RF) & 4 & 20 & 15 \\
\hline
\end{tabular}




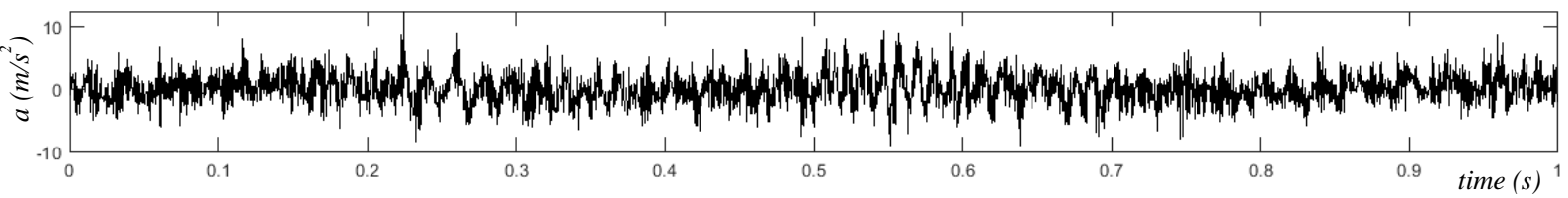

(a)

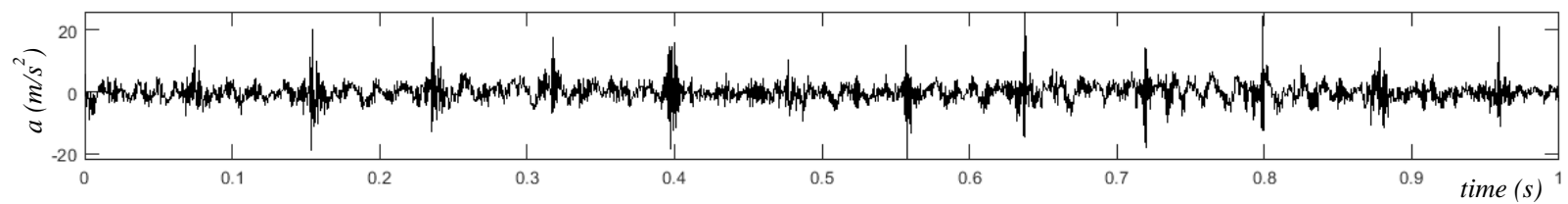

(b)

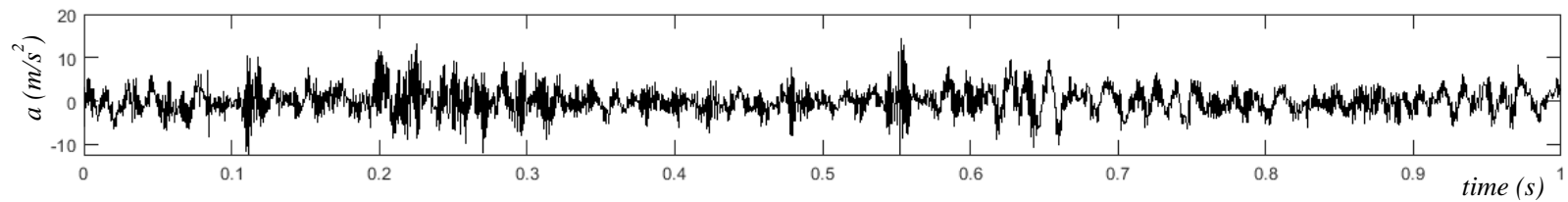

(c)

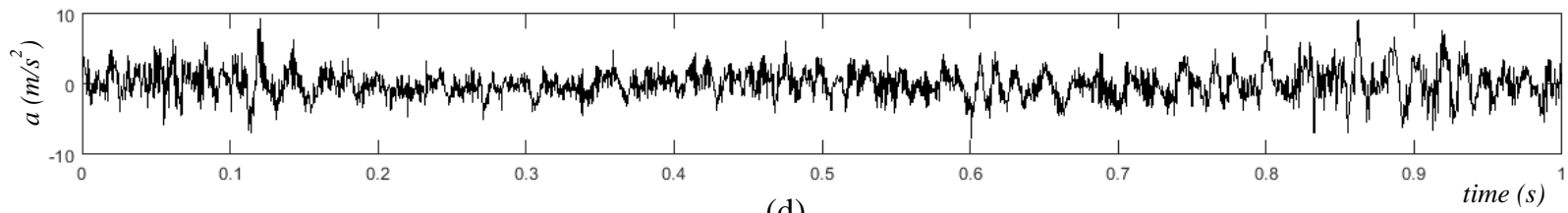

(d)

Figure. 5 Samples of bearing condition: (a) NB singal, (b) IF signal, (c) OF signal, (d) RF signal

with the smallest LaS are used to construct the new sensitive fault feature $F^{\prime}$, which are the most important features and have the strongest ability to distinguish the fault patterns. Correspondingly, the matrix $F$ is optimized as $F^{\prime}$ with dimension 60x5. For the above experimental data, the 30 features are rearranged by LaS as: $\left(\mathrm{LaS}_{9}, \mathrm{LaS}_{3}, \mathrm{LaS}_{11}, \mathrm{LaS}_{6}, \mathrm{LaS}_{5}\right.$, $\mathrm{LaS}_{12}, \mathrm{LaS}_{10}, \mathrm{LaS}_{4}, \mathrm{LaS}_{7}, \mathrm{LaS}_{15}, \mathrm{LaS}_{17}, \mathrm{LaS}_{1}, \mathrm{LaS}_{16}$, $\mathrm{LaS}_{27}, \mathrm{LaS}_{18}, \mathrm{LaS}_{2}, \mathrm{LaS}_{21}, \mathrm{LaS}_{23}, \mathrm{LaS}_{22}, \mathrm{LaS}_{29}, \mathrm{LaS}_{24}$, $\mathrm{LaS}_{26}, \mathrm{LaS}_{28}, \mathrm{LaS}_{8}, \mathrm{LaS}_{30}, \mathrm{LaS}_{13}, \mathrm{LaS}_{25}, \mathrm{LaS}_{19}, \mathrm{LaS}_{20}$, $\mathrm{LaS}_{14}$ ), where LaSi stands for the score of the ith feature in initial feature vector. The above rearranged features indicate that the features $E_{i}=$ $[R M S, S, K, I F, C F, S E]$ of the first five IMFs of vibration signal play more important roles in reflecting the fault information of rolling bearing.

Finally, a set of BSO-LSSVM(i) $i=1,2,3,4$ are designed to identify bearing conditions HB, IR fault, OR fault and RE fault detailed in Table 4. In which, the label of bearing status is assigned to $(+1)$ in the status need to be identified and other labels of bearing

Table 4. Classifier BSO-LSSVM model

\begin{tabular}{lcccc}
\hline \multirow{2}{*}{ Classifier model } & \multicolumn{5}{c}{ Bearing condition } \\
\cline { 2 - 5 } & NB & IF & OF & RF \\
\hline BSO-LSSVM1 & $\mathbf{( + 1 )}$ & $(-1)$ & $(-1)$ & $(-1)$ \\
BSO-LSSVM2 & $(-1)$ & $(+\mathbf{1})$ & $(-1)$ & $(-1)$ \\
BSO-LSSVM3 & $(-1)$ & $(-1)$ & $(+\mathbf{1})$ & $(-1)$ \\
BSO-LSSVM4 & $(-1)$ & $(-1)$ & $(-1)$ & $(+\mathbf{1})$ \\
\hline
\end{tabular}

status are assigned to (-1). The classification result the obtained diagnosis accuracy is $97 \%$ for RB condition which is a difficult status to identify exactly. To demonstrate outperforming of the proposed EEMD-LaS feature selection, we use the initial $F$ feature matrix to train and test the BSO-LSSVM(i) classifier model. The diagnosis result shown in Table 6. Results show that diagnosis accuracy is lower than using the EEMD-LaS feature on the respective diagnosis task and especially consumed the time for training classifier model. Compare the results in Table 5, 6 to emphasize that the redundancy features affect computing results and time-consuming. Therefore, the proposed method for bearing fault diagnosis based on EEMD-LaS - BSO- LSSVM has effectiveness in classification accuracy. The superior quality of the EEMD-LaS features expressed in the optimized classifier BSO-LSSVM model to obtain a significant result.

To compare the diagnosis result, the LSSVM classifier is established using the LibSVM library to perform the classification procedure. We construct the classifiers $\operatorname{LSSVM}(i), i=1,2,3,4$ to identify the rolling bearing conditions. Two feature datasets of the vibtation signal are the original 30-dimensional $F$ feature data and the proposed 5-dimensional $F$, feature data of EEMD-LaS used to train and test the $\operatorname{LSSVM}(i)$, respectively. The evaluation results of LSSVM classifiers are shown in Table 7. In fact, the 
Table 5. Diagnosis result based on EEMD-LaS - BSO-LSSVM

\begin{tabular}{|c|c|c|c|c|c|c|c|c|c|c|}
\hline \multirow[b]{3}{*}{ BSO-LSSVM1 } & \multirow[b]{3}{*}{3.14} & \multirow{2}{*}{\multicolumn{4}{|c|}{ EEMD-LaS Features }} & \multicolumn{2}{|c|}{ Patterns } & \multirow{3}{*}{$\begin{array}{c}\begin{array}{c}\text { Diagnosis } \\
\text { accuracy } \\
(\%)\end{array} \\
100.0\end{array}$} & \multirow{3}{*}{$\begin{array}{c}\begin{array}{c}\text { Time } \\
(\mathbf{s})\end{array} \\
13.20\end{array}$} & \multirow{3}{*}{$\begin{array}{r}\begin{array}{r}\text { Class } \\
\text { task }\end{array} \\
1\end{array}$} \\
\hline & & & & & & \multirow{2}{*}{$\begin{array}{c}\text { Training } \\
60\end{array}$} & \multirow{2}{*}{$\begin{array}{c}\text { Testing } \\
20\end{array}$} & & & \\
\hline & & 2.60 & 4.85 & 7.90 & 3.76 & & & & & \\
\hline BSO-LSSVM2 & 4.41 & 5.02 & 6.39 & 7.6246 & 7.96 & 60 & 20 & 97.33 & 5.13 & 2 \\
\hline BSO-LSSVM3 & 8.15 & 10.12 & 7.75 & 7.0366 & 9.12 & 60 & 20 & 100.0 & 5.15 & 3 \\
\hline BSO-LSSVM4 & 5.23 & 4.35 & 6.69 & 7.6632 & 6.07 & 60 & 20 & 97.0 & 6.17 & 4 \\
\hline
\end{tabular}

Table 6. Diagnosis result based on initial feature

\begin{tabular}{|c|c|c|c|c|c|}
\hline & \multicolumn{2}{|c|}{ Patterns } & \multirow{2}{*}{$\begin{array}{c}\text { Diagnosis } \\
\text { accuracy } \\
(\%)\end{array}$} & \multirow{2}{*}{$\begin{array}{c}\text { Time } \\
\text { (s) }\end{array}$} & \multirow{2}{*}{$\begin{array}{l}\text { Class } \\
\text { task }\end{array}$} \\
\hline & Training & Testing & & & \\
\hline BSO-LSSVM1 & 60 & 20 & 93.5 & 23.30 & 1 \\
\hline BSO-LSSVM2 & 60 & 20 & 87.33 & 71.23 & 2 \\
\hline BSO-LSSVM3 & 60 & 20 & 89.0 & 21.74 & 3 \\
\hline BSO-LSSVM4 & 60 & 20 & 85.0 & 68.19 & 4 \\
\hline
\end{tabular}

Table 7. Diagnosis result based on LSSVM

\begin{tabular}{|c|c|c|c|c|c|c|c|}
\hline & \multicolumn{2}{|c|}{ Patterns } & \multicolumn{2}{|c|}{ EEMD } & \multicolumn{2}{|c|}{ EEMD-LaS } & \multirow[b]{2}{*}{ Class task } \\
\hline & Training & Testing & $\begin{array}{c}\text { Diagnosis } \\
\text { accuracy } \\
(\%)\end{array}$ & $\begin{array}{c}\text { Time } \\
\text { (s) }\end{array}$ & $\begin{array}{c}\text { Diagnosis } \\
\text { accuracy } \\
(\%)\end{array}$ & $\begin{array}{c}\text { Time } \\
\text { (s) }\end{array}$ & \\
\hline LSSVM 1 & 60 & 20 & 65 & 1.218 & 85 & 1.196 & 1 \\
\hline LSSVM 2 & 60 & 20 & 53 & 0.894 & 73 & 0.878 & 2 \\
\hline LSSVM 3 & 60 & 20 & 50 & 1.009 & 80 & 0.943 & 3 \\
\hline LSSVM 4 & 60 & 20 & 65 & 1.281 & 75 & 1.031 & 4 \\
\hline
\end{tabular}

diagnosis accuracy results in the table shown that evaluation on the conventional LSSVM classifiers obtained the very poor accuracy. The highest diagnosis accuracy is only $85 \%$, it is lower than the accuracy of the our proposed BSO-LSSVM in the same normal bearing condition. Thus, this diagnosis model usually tends to produce a rejection and not reuse. The comparison on two feature dataset of $F$ and $F^{\prime}$, the proposed EEMD-LaS method extracted the effected feature in low-dimensional data to gain the higher classification accuracy even the LSSVM classifiers. Nevertheless, the perfection of the proposed BSO-LSSVM in the diagnosis accuracy results paid the higher price for time than the conventional LSSVM model. The cause of this problem is that the BSO-LSSVM model is always optimized in constructing model.

\section{Conclusions}

This study proposes the diagnosis method for four conditions of rolling bearing $\mathrm{HG}$, IF, OF, and $\mathrm{RB}$ based on the compact sensitive feature of EEMD-LaS and optimized BSO-LSSVM classifier model, EEMD-LaS - BSO-LSSVM. The obtained diagnosis result based on experimental data is the significance values. The identification accuracy of NB, OR conditions of the rolling bearing are identified exactly $100 \%$, and $97 \%$ identify even the difficult condition of bearing RF fault with a low time cost. The compact sensitive EEMD-LaS features are firstly explored by the LaS algorithm on the initial features of the basic EEMD method. The LaS measures feature acording to the order of influence of the internal fault information. These EEMD-LaS features are fed into the optimized BSO-LSSVM classifier model as the input for performing automatic identification. BSO algorithm is employed to address the shortcomings of the manual setting parameter of the LSSVM classifier to generate the search-direction matrix for the next iteration to obtain the best LSSVM performance. To can further study in bearing faults diagnosis, more features are extracted based on EEMD-LaS to provide enhanced recognition performance.

\section{Conflicts of Interest}

The authors declare that they have no conflict of interest.

\section{Author Contributions}

Author 1: Investigation, resources, make contributions to conception and design, writing- 
review and editing, analysis, and interpretation of data.

Author 2: Collect the data, visualization, methodology, data curation, writing-original draft preparation

Author 3: Participate in drafting the article, supervision and project administration

\section{Acknowledgments}

The authors would like to express our gratitude to Faculty of Mechanical Engineering, Hanoi University of Industry.

\section{References}

[1] Z. Wu and N. E. Huang, "Ensemble empirical mode decomposition: a noise-assisted data analysis method", Advances in Adaptive Data Analysis, Vol. 01, pp. 1-41, 2009.

[2] N. E. Huang, Z. Shen, S. R. Long, M. C. Wu, H. H. Shih, Q. Zheng, N. C. Yen, C. C. Tung, and H. H. Liu, "The empirical mode decomposition and the Hilbert spectrum for nonlinear and nonstationary time series analysis", In: Proc. of the Royal Society of London. Series A: Mathematical, Physical and Engineering Sciences, Vol. 454, p. 903, 1998.

[3] K. Fang, H. Zhang, H. Qi, and Y. Dai, "Comparison of EMD and EEMD in rolling bearing fault signal analysis", In: Proc. of 2018 IEEE International Instrumentation and Measurement Technology Conference (I2MTC), pp. 1-5, 2018.

[4] V. H. Nguyen, J. S. Cheng, Y. Yu, and V. T. Thai, "An architecture of deep learning network based on ensemble empirical mode decomposition in precise identification of bearing vibration signal", Journal of Mechanical Science and Technology, Vol. 33, pp. 41-50, 2019.

[5] T. Kim, J. Y. Shin, S. Kim, and J. H. Heo, "Identification of relationships between climate indices and long-term precipitation in South Korea using ensemble empirical mode decomposition", Journal of Hydrology, Vol. 557, pp. 726-739, 2018.

[6] L. Song, A. Smola, A. Gretton, K. M. Borgwardt, and J. Bedo, "Supervised feature selection via dependence estimation", In: Proc. of the 24th international conference on Machine learning, pp. 823-830, 2007.

[7] X. He, D. Cai, and P. Niyogi, "Laplacian score for feature selection", Advances in neural information processing systems, Vol. 18, pp. 507-514, 2005.
[8] L. Zhu, L. Miao, and D. Zhang, "Iterative Laplacian score for feature selection", In: Proc. of Chinese conference on pattern recognition, 2012, pp. 80-87.

[9] S. Wold, K. Esbensen, and P. Geladi, "Principal component analysis", Chemometrics and Intelligent Laboratory Systems, Vol. 2, pp. 3752, 1987.

[10] X. Y. Jing, S. Li, D. Zhang, J. Yang, and J. Y. Yang, "Supervised and unsupervised parallel subspace learning for large-scale image recognition", IEEE Transactions on Circuits and Systems for Video Technology, Vol. 22, pp. 1497-1511, 2012.

[11] J. A. K. Suykens and J. Vandewalle, "Least Squares Support Vector Machine Classifiers", Neural Processing Letters, Vol. 9, pp. 293-300, 1999.

[12] S. Abbasion, A. Rafsanjani, A. Farshidianfar, and N. Irani, "Rolling element bearings multifault classification based on the wavelet denoising and support vector machine", Mechanical Systems and Signal Processing, Vol. 21, pp. 2933-2945, 2007.

[13] K. C. Gryllias and I. A. Antoniadis, "A Support Vector Machine approach based on physical model training for rolling element bearing fault detection in industrial environments", Engineering Applications of Artificial Intelligence, Vol. 25, pp. 326-344, 2012.

[14] K. W. Lau and Q. H. Wu, "Local prediction of non-linear time series using support vector regression", Pattern Recognition, Vol. 41, pp. 1539-1547, 2008.

[15] B. Long, S. Tian, Q. Miao, and M. Pecht, "Research on features for diagnostics of filtered analog circuits based on LS-SVM", in Autotestcon, pp. 360-366, 2011.

[16] S. Li, W. Zhou, Q. Yuan, S. Geng, and D. Cai, "Feature extraction and recognition of ictal EEG using EMD and SVM", Comput Biol Med, Vol. 43, pp. 807-16, 2013.

[17] Y. Gao, X. Wang, Y. Cheng, and J. Pan, "Fault diagnosis using a probability least squares support vector classification machine", Mining Science and Technology, Vol. 20, pp. 917-921, 2010.

[18] Z. Su, B. Tang, Z. Liu, and Y. Qin, "Multi-fault diagnosis for rotating machinery based on orthogonal supervised linear local tangent space alignment and least square support vector machine", Neurocomputing, Vol. 157, pp. 208222, 2015.

[19] P. Civicioglu, "Backtracking search optimization algorithm for numerical 
optimization problems", Applied Mathematics and computation, Vol. 219, pp. 8121-8144, 2013.

[20] Y. Lei, J. Lin, Z. He, and M. J. Zuo, “A review on empirical mode decomposition in fault diagnosis of rotating machinery", Mechanical Systems and Signal Processing, Vol. 35, pp. 108-126, 2013.

[21] L. Jinshan and C. Qian, "Application of the EEMD method to multiple faults diagnosis of gearbox", In: Proc. of 2010 2nd International Conference on Advanced Computer Control, 2010, pp. 395-399.

[22] V. Nguyen, T. D. Hoang, V. Thai, and X. Nguyen, "Big Vibration Data Diagnosis of Bearing Fault Base on Feature Representation of Autoencoder and Optimal LSSVM-CRO Classifier Model", In: Proc. of 2019 International Conference on System Science and Engineering (ICSSE), pp. 557-563, 2019. 